\title{
Re-operative Single Incision Laparoscopic Surgery: A Feasible Surgical Option in Highly Selected Clinical Setting
}

\author{
Duck-Woo Kim, M.D., Ph.D. \\ Department of Surgery, Seoul National University Bundang Hospital, Seongnam, Korea
}

\author{
Corresponding author \\ Duck-Woo Kim \\ Department of Surgery, Seoul National University Bundang Hospital, 82, Gumi-ro 173 Beon-gil, Bundang-gu, Seongnam 13620, Korea \\ Tel: +82-31-787-7101, Fax: +82-31-787-4055, E-mail: kdw@snubh.org \\ ORCID: http://orcid.org/0000-0001-9218-4676
}

This is an Open Access article distributed under the terms of the Creative Commons Attribution Non-Commercial License (http:/ creativecommons.org/licenses/by-nc/4.0/) which permits unrestricted non-commercial use, distribution, and reproduction in any medium, provided the original work is properly cited.

Copyright $\odot 2018$ The Journal of Minimally Invasive Surgery. All rights reserved.
Over the past decade, surgeons tried to find a less invasive surgical approach method compared to multi-port laparoscopic surgery to improve short-term outcomes. These efforts lead to finding newer methods, such as natural orifice transluminal surgery or single-incision laparoscopic surgery (SILS). To date, there have been many favorable studies reporting the potential benefits of SILS over multi-port laparoscopic surgery in terms of less postoperative pain, reduction of morbidity, better cosmetic outcome, and better overall patient satisfaction. ${ }^{1-3}$

In spite of previous studies, the true benefits of SILS over multi-port laparoscopic approach in major colorectal surgery remain unclear. Moreover, the obvious complexity in manipulating various surgical instruments through a single port requires an adequate learning period. The present study reported the short-term results of re-operative SILS (R-SILS) based on a large patient cohort that underwent primary SILS for colorectal diseases. The authors reported comparative outcomes of R-SILS to multi-port laparoscopic surgery in terms of postoperative complications and length of hospital stay. They also showed R-SILS could be applied to both elective and emergent operations.

As the authors comment as a limitation of this study, all RSILS were performed by only one fully-experienced surgeon. R-SILS in patients with postoperative complications will defi- nitely require a more proficient set of surgical skills. Currently available literatures on SILS in colorectal surgery have certain limitations that need to be clarified. ${ }^{1}$ First, most of the studies were retrospective in nature and based on highly selective patients. ${ }^{4}$ The studies were also very heterogeneous in the range of surgical procedures, equipment/devices used, and patients' characteristics. ${ }^{5}$ Most importantly, SILS is usually performed by highly motivated groups with good SILS skills. So, these favorable results may not be reproducible by the other surgeons. A recent meta-analysis based on 32 studies including 3,863 patients excellently summarized the current status of SILS. In this review, colorectal SILS had comparative outcomes to multi-port laparoscopic surgery in terms of operative time, conversion rate, postoperative complications and mortality. The short-term oncological results of SILS for colorectal cancer were satisfactory including lymph node harvest rates and resection margins. Nevertheless, there are currently no available long-term results comparing the survival rates and recurrence rates.

SILS and R-SILS performed by proficient SILS surgeons may have comparative results to multi-port laparoscopic colorectal surgery. In this setting, SILS could be a feasible and safe surgical option in re-operative colorectal surgery with good surgical outcomes for selected patients. However, to 
date, the evidences are far too insufficient to recommend SILS as a widespread approach replacing multi-port laparoscopic surgery, even more so in re-operative settings. Results from presently conducted large RCTs are needed to establish the indications and efficacy for SILS in colorectal surgery.

\section{REFERENCES}

1) Hoyuela C, Juvany M, Carvajal F. Single-incision laparoscopy versus standard laparoscopy for colorectal surgery: A systematic review and meta-analysis. Am J Surg 2017;214:127-140.

2) Fung AK, Aly EH. Systematic review of single-incision laparoscopic colonic surgery. Br J Surg 2012;99:1353-1364.
3) Lujan JA, Soriano MT, Abrisqueta J, Perez D, Parrilla P. Singleport Colectomy VS Multi-port Laparoscopic Colectomy. Systematic Review and Meta-analysis of More Than 2800 Procedures. Cir Esp 2015;93:307-319.

4) Rosati CM, Boni L, Dionigi G, et al. Single port versus standard laparoscopic right colectomies: results of a case-control retrospective study on one hundred patients. Int J Surg 2013;11 Suppl 1:S50-53.

5) Pedraza R, Aminian A, Nieto J, Faraj C, Pickron TB, Haas EM. Single-incision laparoscopic colectomy for cancer: shortterm outcomes and comparative analysis. Minim Invasive Surg 2013;2013:283438. 